\title{
Numerical study of the effect of explosive sources near the ground surface: crater formation
}

\author{
Ignacio Iturrioz ${ }^{(1)}$ and Jorge D. Riera ${ }^{(2)}$ \\ (1) PROMEC, Dept. of Mechanical Engineering, School of Engineering, UFRGS, Porto Alegre, RS, Brazil \\ e-mail:ignacio@mecanica.ufrgs.br \\ (2) PPGEC, Dept. of Civil Engineering, School of Engineering, UFRGS, Porto Alegre, RS, Brazil \\ e-mail: riera@cpgec.ufrgs.br
}

\begin{abstract}
In this paper.. results of numerical evaluations of the response of the ground to explosive charges located above the ground surface are presented and compared with experimental observations. The crater dimensions and the size of the damaged volume are estimated using a so-called discrete element representation (DEM) of the medium, employed earlier by the authors in fracture and impact response studies, together with well known equations to define the blast pressures. It is shown that the predictions are consistent with measured response, and that the approach may therefore be used with confidence in engineering design.
\end{abstract}

\section{INTRODUCTION}

The effects of blasting loads or explosions of flammable products must be taken into account in the design of NPPs whenever the probability of occurrence of such events exceeds established thresholds. In the last two decades, structural design against the resulting pressure waves or against projectiles caused by the explosion, has registered considerable progress and, in some cases, it has also been validated through experimental verification. On the other hand, there is scant information on the response of the ground to explosions on or above the soil surface, which may form craters and induce damage in buried ducts, tanks or other structures and constitutes the central topic of this paper.

Dynamic loads due to explosions cause large deformations, with strain rates ranging from $10^{-1}$ to $10^{-3} \mathrm{~s}^{-1}$, which implies short time dynamic behavior of the materials, commonly characterized by a great over-strength and a less pronounced stiffness increase, in comparison with static properties. In the case of soils, prediction of the response as well as the mechanism of crater formation are still more complex, due to the inherent nonlinear material behavior, to the nonhomogeneity that characterizes most soil sites and to the coexistence of three phases: solid, liquid and gaseous. Hence, drastic simplifications must be made in order to solve specific problems for engineering purposes. In this context, note that most available solutions for practical problems have been obtained through purely empirical approaches, condensed in design charts or equations (Baker et al, [ 2], Smith \& Hetherington, [ 8])

In connection to soils response, most research is related to underground explosions, with only a few papers concerned with explosions at ground level. Neither experimental nor theoretical studies about craters produced by explosions above ground level have been found in the technical literature. This would be the case when the explosive charge or the gas cloud are situated at some distance from the ground surface, and has been a matter of interest in connection with a number of design situations encountered by the authors in recent years. By way of introduction, a summary of experimental studies on crater formation due to blast loading is presented in the following section, including a brief description of the results obtained in the first stage of an experimental program conducted jointly by UNT, Argentina and UFRGS, Brazil (Ambrosini et al, [ 1]). Numerical analysis of the problem may be attempted with commercial Finite Element programs, such as. LSDYNA (2000) or ABAQUS (1997), but both present the limitation that the formation of the full system stiffness matrix is required, which renders any possible fracturing or fragmentation of the medium almost untractable. On the other hand, the so-called Discrete Element method (DEM) constitutes an attractive alternative, allowing the consideration of fracturing and fragmentation without difficulty. The approach has been previously employed by the authors to determine the response of elastoplastic shells to blasting loads (Riera \& Iturrioz, [ 6]) and the fracture behavior of concrete structures subjected to impact (Riera \& Iturrioz, [ 7]), and is herein applied to the study of crater formation and damage distribution in soils subjected to explosions above the ground. 


\section{EXPERIMENTAL STUDIES ON CRATER FORMATION DUE TO BLAST LOADING}

Tests of crater formation are appropriate tools to study the behavior and destructive power of different explosives and the response of soils and rocks to this type of load (Persson et al, 1994). However, the mechanism of crater formation is complex and the geometrical and physical properties of the systems under consideration difficult to define without large experimental error, so even very carefully performed crater tests yield deviations in the measured dimensions of the order of $10 \%$, while differences as large as 30 or $40 \%$ are not uncommon (Bull et all, [ 3])

Studies concerned with the characteristics of craters caused by explosions usually resort to dimensional analysis. In fact, the scaling laws establish that any linear dimension $L$ of the crater can be expressed as a constant multiplied by $W^{1 / 3}$, in which $W$ denotes the mass of explosive (TNT or TNT equivalent), expressed in the following in $\mathrm{kg}$. A cavity is always formed when a confined explosion is produced in a mass of soil. If the explosive charge is close to the surface, a crater is formed. The two most important variables in defining the response of the system are the mass $W$ and the distance $d$ of the explosive charge to the air-soil interface, considered herein negative when the charge is located above the surface, and positive in case of underground explosions. For $d>0$, the crater formation is influenced by gravity. Since as the depth of the detonation increases, larger amounts of subsoil must be expelled by the explosion, the crater radius and depth increase as $d$ increases, until a limit value is reached, after which both radius and depth rapidly decrease. Let:

$$
\eta=d / W^{1 / 3}
$$

According to Bull et all [ 3], the largest crater is formed when $1.0<\eta<1.5$, while in the range $-0.5<\eta<2.0$, a conventionally shaped crater is formed. Baker et all [ 2] present a dimensional study to model the crater formation phenomenon in underground explosions. Six parameters are chosen to define the problem: the weight of explosive $W$, the depth of the charge $d$, the apparent crater radius $R$, the soil density $\rho$, a seismic wave velocity $c$ and the acceleration of gravity $g$ (Ambrosini et all, [ 1]), which enter into an adimensional term $\Pi$ :

$$
\left.\Pi=\left[W^{7 / 24} \rho^{-7 / 24} c^{-1 / 3} g^{-1 / 8} d^{-1}\right)\right]
$$

When experimental results for different types of soil are evaluated, relating $D / d$ with $\Pi, D$ denoting the crater diameter, it is readily seen that they present little variability. The preceding comments refer to underground explosions. There is less information about explosions at ground level. Regression studies of about 200 accidental superficial explosions are presented by Kinney and Graham [ 4], leading to the crater diameter:

$$
D / W^{1 / 3}=0.8
$$

Experimental results reported by Ambrosini et al (2000), led to the following regression equation:

$$
D / W^{1 / 3}=0.60+1.50 \eta+2.10 \eta^{2}-0.75 \eta-1.70 \eta^{4}
$$

Which satisfactorily fits the data obtained in a single testing site, in the range $-1<\eta<1$. Note that for $\eta=0$, eq.(4) predicts crater diameters about $25 \%$ smaller than expression (3). The difference is indicative of the variability of empirical prediction equations for crater sizes. Finally, it should be mentioned that no indication was found in the literature on the dimensions of the ground region fractured, damaged, or otherwise affected by the explosion, which obviously extends beyond the crater region, nor on the vibrations induced in the surrounding medium.

\section{THE DISCRETE ELEMENT METHOD: BASIC FEATURES AND PREVIOUS APPLICATIONS}

The computational method employed is based on the representation of a continuum by means of an array of nodal masses interconnected by one-dimensional elements without mass. The cubic array shown in fig 1 , consisting of a cubic cell with eight nodal points at the vertices plus a central node, each with three degrees of freedom, was adopted. The masses are linked by longitudinal and diagonal elements of lengths $L_{c}$ and $\sqrt{3} / 2 L_{c}$, respectively. The equivalence between an orthotropic elastic solid with the principal material axes oriented in the direction of the longitudinal elements and the cubic lattice model was clearly demonstrated (Riera \& Iturrioz, [6], [7]) within the framework of linear elasticity: Only a restriction on the value 
of Poisson's ratio must be imposed for a complete equivalence $(v=0.25)$. For other values of $v$ the model results in minor differences in the shear terms. The equations of motion of the resulting $\mathrm{N}$ DOF system may be expressed in the form:

$$
\boldsymbol{M} \overrightarrow{\ddot{u}}+\vec{f}(t)=\vec{Q}(t)
$$

In which $\boldsymbol{M}$ denotes the (diagonal) mass matrix, $\vec{u}$ the vector of generalized coordinates (nodal displacements), $f(t)$ the internal nodal forces, which may depend on present (elastic) and past nodal displacements, and $Q(t)$ the externally applied forces. Thus, in linear elastic systems, $f(t)=K u, K$ being the stiffness matrix. In a system with linear viscous forces, $f(t)=K u+$ $C \dot{u}, C$ is assumed to be proportional to the mass matrix, the system (5) may be numerically integrated in the time domain by means of the explicit central finite difference algorithm. By updating the nodal coordinates at each time step, the approach allows the consideration of large displacements, i. e. geometrical non-linearity. The convergence of the solutions in linear elasticity, as well as in elastic instability problems, was also verified by the authors. More recently, Rocha and Riera [ 8], extended the method to fracture analysis of brittle structures, such as concrete or cohesive soils. The stress-strain or forcedisplacement relationship for the material is given by a triangular diagram, as shown in fig 2 . The limit strain $\varepsilon_{\mathrm{r}}$ is chosen to satisfy the condition that when an element fails and a crack opens, an amount of energy is dissipated. This energy is equal to the product of the ruptured surface area, which is related to $L_{c}$, times the critical surface energy $G_{f}$ of the material. A second assumption is that material properties may be modelled as random fields, i.e. vary from element to element.

\section{EXPLOSIVE CHARGE ON A HOMOGENEOUS HALF-SPACE}

\section{Pressures induced by an explosive charge}

The evolution of blast pressures with time in case of explosions in air has been extensively studied and can be described with reasonable confidence (Kinney \& Graham [4]). Assuming a point charge of TNT mass W (or TNT equivalent mass), and denoting as $r$ the distance from any location of interest to the point charge, a scaled distance $z$ is defined as:

$$
z=r / W^{1 / 3}
$$

The relation between the pressure at the location of interest is given by the expression

$$
P=p_{s}\left(1-t / t_{d}\right) \exp \left(-\alpha t / t_{d}\right)
$$

In which $p$ is the peak overpressure at the poimt of interest at time $t, t$ denotes the time measured from the instant when the pressure wave reaches the point, and $t_{d}$ is the duration of the triangular pulse. The wave form parameter $\alpha$ in eq (7) may be regarded as an adjustable factor, selected so that the overpressure-time relations provide suitable values of blast impulse. Figure 3 shows a plot of the variation of pressure wwith time. The three parameters $p_{s,} t_{d}$ and $\alpha$ depend on $z$, and may be found in tables (Kinney \& Graham, [ 4]) to facilitate computation. Finally, the wave velocity $c_{s}$ is given by the equation :

$$
c_{s}=c_{o} M(z)
$$

in which $c_{o}$ denotes the pressure waves (sound) velocity in air, while $M$ is Mach's number. The variation of $M$ with $z$ may also be found in Kinney and Graham [4].

\section{Discrete Model of a half-space}

The discrete element method (DEM) described in the previous section was used to model a region of a half space . On account of symmetry and to reduce the numerical effort, only a block bounded by planes $\mathrm{x}=0$ and $x=3 m, y=0$ and $y=3 m$, $z=0$ and $z=3 m$ was considered. Since the objective was to model a half space, transmitting boundaries were introduced at $x=y=z=3 m$. The element size was taken equal to $0.1 \mathrm{~m}$, which implies restricting the volume of the smallest "chunk" of soil material expelled in the process of crater formation to $10^{-3} \mathrm{~m}^{3}$. This is too large, since in the experimental studies (Ambrosini 'et $a l^{\prime},[1]$ ), masses of about a tenth of that value were thrown at distance of up to $100 \mathrm{~m}$, not counting finer particles in dust form, for charges located on the surface and at distances of $0.50 \mathrm{~m}$ and $1.00 \mathrm{~m}$, above and below the surface. The model analyzed contains 27 cubic element, 60000 bars, totaling more than 150000 DOF. Consequently, increasing the number of DOF, by reducing the element size $\mathrm{L}_{\mathrm{c}}$ even further, was not feasible at this stage. 
The soil, a stiff clay with some sand and silt, was characterized by $\rho_{o}=1400 \mathrm{~kg} / \mathrm{m}^{3}$, an angle of internal friction $\phi=25^{\circ}$ and cohesion $c=0.036 \mathrm{Mpa}$. The material properties needed for the numerical analysis were inferred from those values, resulting in the following parameters: specific fracture energy $G_{f}=100 \mathrm{~N} / \mathrm{m}$, critical strain $\varepsilon_{\mathrm{p}}=6.6 \times 10^{-4}$ and Young's modulus at the origin: $E=188.0 E 06 \mathrm{~N} / \mathrm{m}^{2}$. The constitutive equation for the elements is defined by

$$
F_{i}=\varepsilon(E A)+\dot{\varepsilon}(A \beta)
$$

where $\mathrm{A}$ is the cross section of the elements and $\varepsilon$ the axial strain. $\mathrm{E}$ varies with $\varepsilon$, as shown in Figure 2 . The parameter $\beta$ characterizes the viscoelastic behavior of the material, i.e. internal damping not accounted for by the energy dissipated by fracture. In the numerical studies, a value $\beta=0.001$, roughly equivalent to a critical damping ratio of $5 \%$ was used. Certainly additional studies are necessary to correctly model energy dissipation within the soil mass.

\section{COMPARISON WITH EXPERIMENTALLY-BASED PREDICTIONS}

In the tests reported by Ambrosini 'et al' [ 1], charges were located in the air at various distances from the ground surface. The effect of these air blasts on the ground was numerically determined in order to assess the performance of the analytical models described above, giving particular emphasis to the crater size. The model of the half-space as well as the blast pressure determination are described above. Results for one of the numerical simulations are presented next (charge W=1kg, $0.01 \mathrm{~m}$ above the surface). Fig. 6 shows the distribution of damage at $\mathrm{t}=97.6 \mathrm{~ms}$ after the explosion, identifying completely cracked elements and merely damages ones. Most nodal masses above $z=3.00 \mathrm{~m}$ are already loose elements, moving away from the crater in formation. The effect may be more clearly seen in Fig. 7, which indicates the position of nodal elements at $t=97.6 \mathrm{~ms}$. The diameter of the future crater may be obtained from this graph or from Fig. 10, which presents the surface profile along a radial plane. The evolution of strain, fracture and kinetic energy with time after the explosion is shown in Figs. 8. In Figure 9, the variation of the vertical and radial accelerations at diferent distance of center blast is shown. Finally, Fig. 10 presents a summary of the comparison between numerical and experimental predictons for the crater diameter. The correlation for the cases studied so far is satisfactory. To determine the ground response for charges located above $0.5 \mathrm{~m}$, a finer mesh appears to be necessary, situation that was not addressed in this paper.

\section{CONCLUSIONS}

A numerical approach for the determination of crater size and damaged region caused by explosive charges above the ground surface has been presented and compared with experimental evidence and empirical prediction equations. Those initial results confirm the applicability and global reliability of the approach for the analysis of structural systems subjected to impact and impulsive loading.

\section{REFERENCES}

[ 1] Ambrosini, R.D.; Luccioni, R.F.; Danesi, R.F.; Riera, J.D. and Rocha, M.M. (2000): "Craters produced by explosions", to be published.

[ 2] Baker, W.E., Cox, P.A., Westine, P.S., Kulesz, J.J. and Strehlow, R.A. (1983): Explosion Hazards and Evaluation, Elsevier, Amsterdam, The Netherlands.

[ 3] Bull, J.W. and Woodford, C.H. (1998): "Camouflets and their effects on runway supports", Computer and Structures, 69/6, 695-706.

[ 4] Kinney, G.F. and Graham, K.J. (1985): Explosive Shocks in Air, Second Edition, Springer Verlag, Berlin.

[ 5] Persson, P.A.; Holmberg, R. and Lee, J.: (1994): Rock blasting and explosives engineering, CRC Press, Boca Raton , USA.

[ 6] Riera, J.D. and Iturrioz, I. (1995): "Discrete element dynamic response of elastoplastic shells subjected to impulsive loading", Communications in Numerical Methods in Engineering, Vol. 11, 417-426.

[ 7] Riera, J.D. and Iturrioz, I.(1998): "Discrete element model for evaluating impact and impulsive response of reinforced concrete plates and shells subjected to impulsive loading", Nuclear Engineering and Design, Elsevier, Amsterdam, The Netherlands, 179, 135-144.

[ 8] Rocha, M.M \& Riera, J.D.: (1990) "On size effects and rupture in non-homogeneous materials", Proceedings, Congress on Fracture Processes in Concrete, Rock and Ceramics, J.M.Van Mier; J.G. Rots \& A. Baker (Eds), Chapman \& Hall/ Ed. Fn Spon, London, 451-460. 
[ 9] Smith, P.D. and Hetherington (1994): Blast and Ballistic Loading of Structures, Butterworth-Heinemann Ltd., London, U.K.

\section{ACKNOWLEDGEMENTS}

The writers wish to acknowledge the partial support of CNPQ, Brazil. Calculations were perfomed at CESUP,UFRGS.
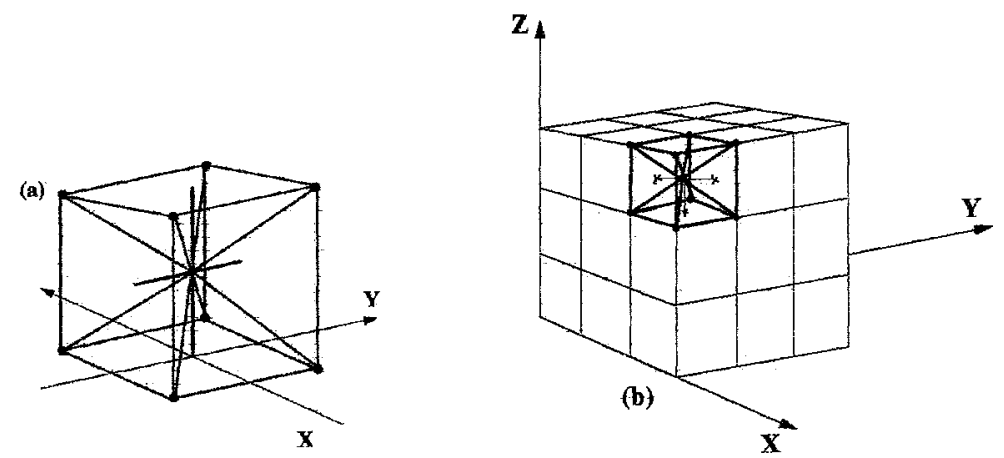

figure 1: Cubic array (a): a basic module, (b) Composition of prisms.
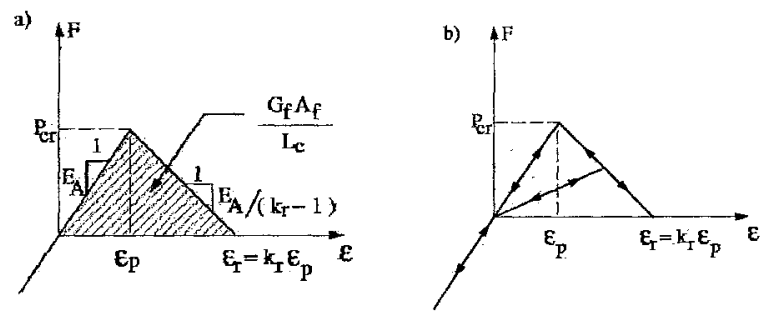

Figure 2: Constitutive bilinear law of brittle material

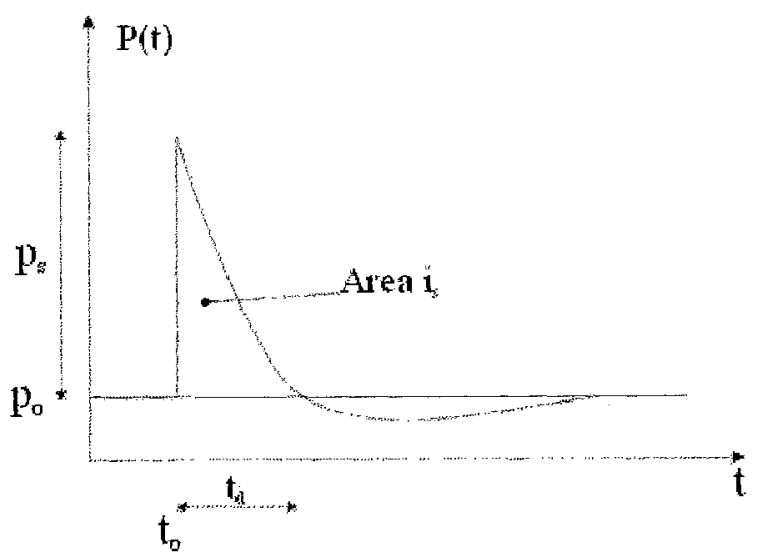

Figure 3: Typical pressure-time curve for na explosive blast wave 

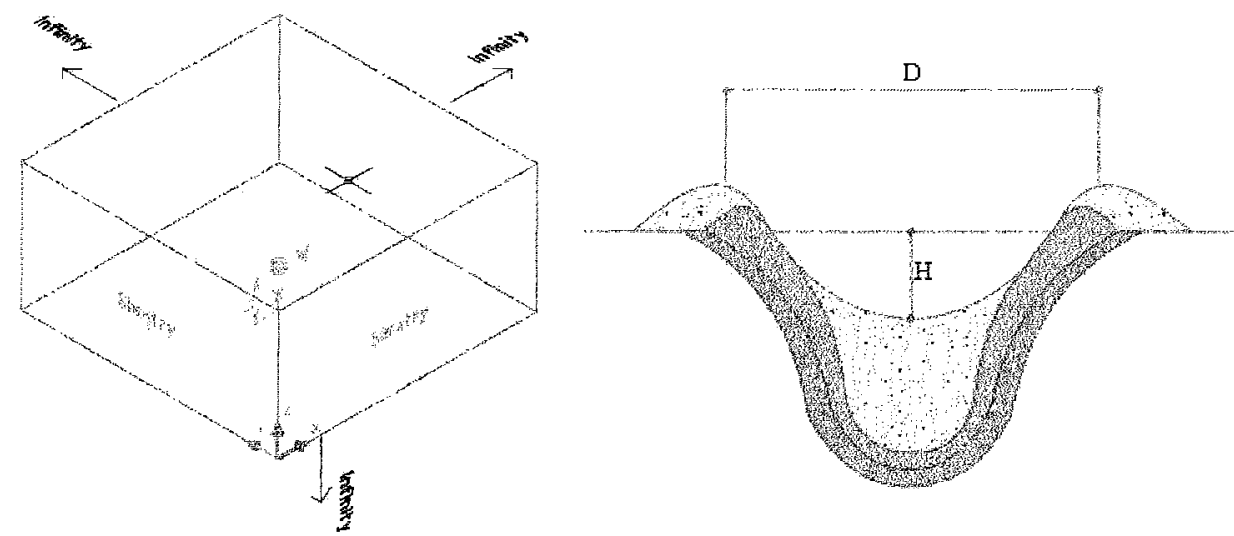

Figure 4: a)Layout of discretized half space model b) esquematical representation of blast crater in the half space.

Lateral View

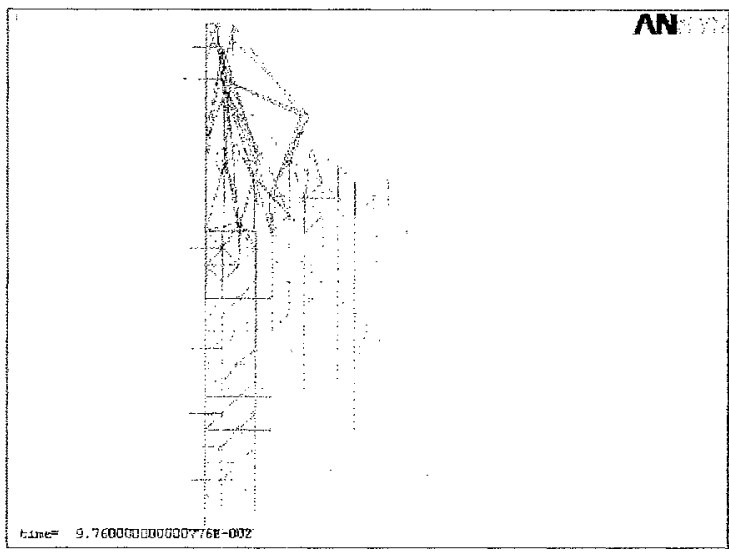

Frontal View

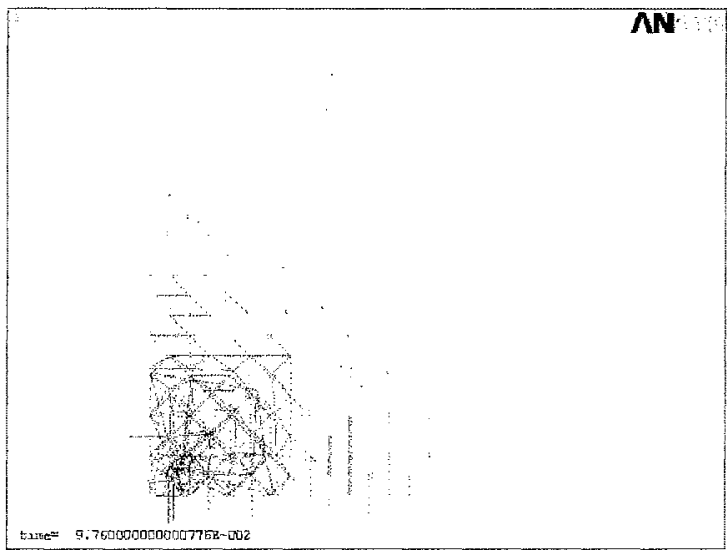

Figura 6: Configuration of fractured and damaged bars in numerical simulation with $\mathrm{W}=1 \mathrm{~kg}$, and the explosive charge on the ground surface $\left(h_{0}=0.01 \mathrm{~m}\right)$. a)only fractured bars, $\mathrm{b}$ ) fractured and damaged bars. ( fractured bars:red, damaged bars: yellow.)

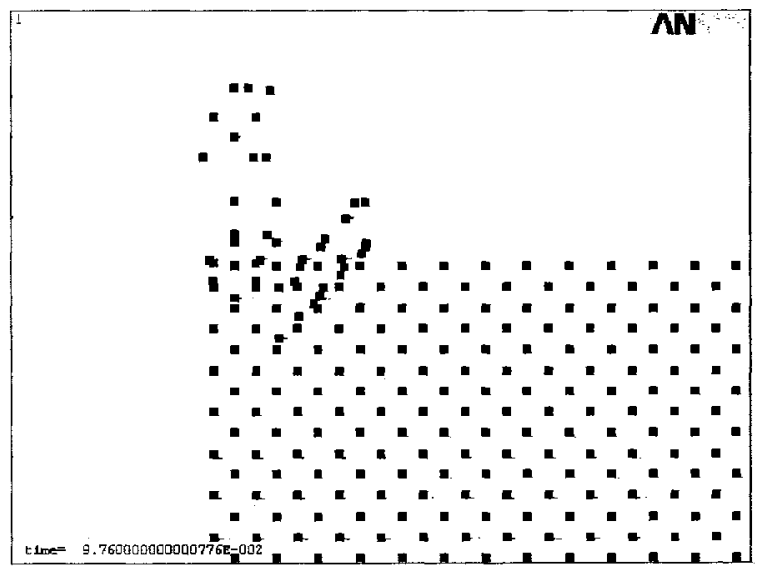

Figure 7. Deformed configuration at $97.6 \mathrm{~ms}$ for the numerical simulation with $\mathrm{W}=1 \mathrm{~kg}$ and The explosive charge on the ground surface $\left(\mathrm{h}_{0}=0.01 \mathrm{~m}\right)$. 


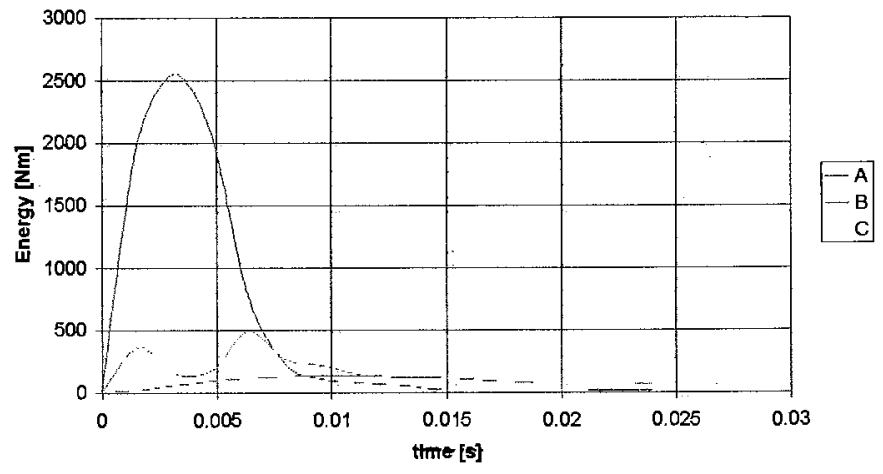

Figura 8: Energy vs Time according to numerical simulation with $\mathrm{W}: 1 \mathrm{~kg}$, and explosive charge on the ground surface. (A:Elastic strain energy, B: Fracture Energy, C: Kinetic Energy).

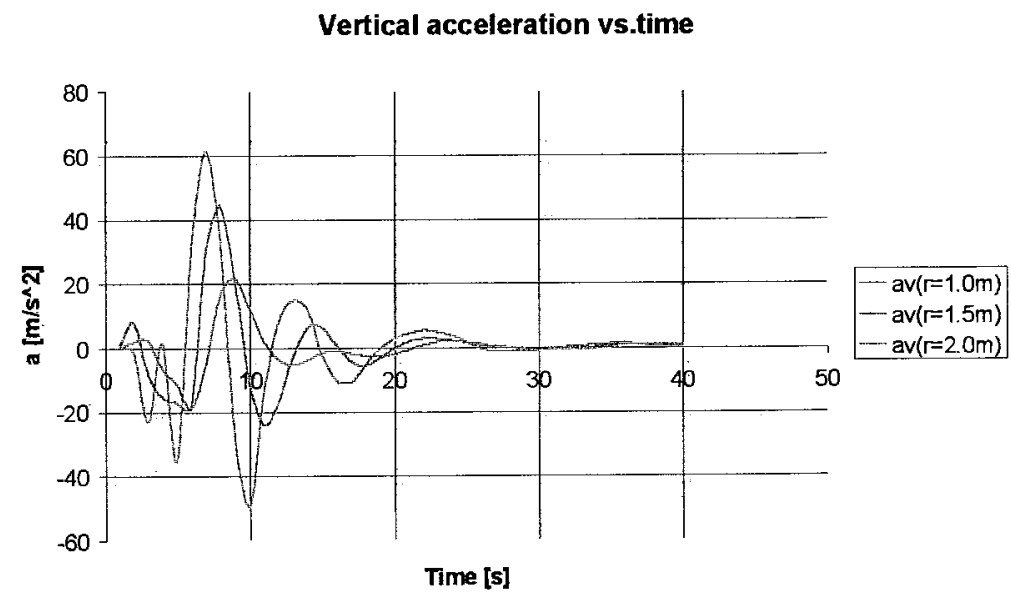

Radial acceleration vs. time

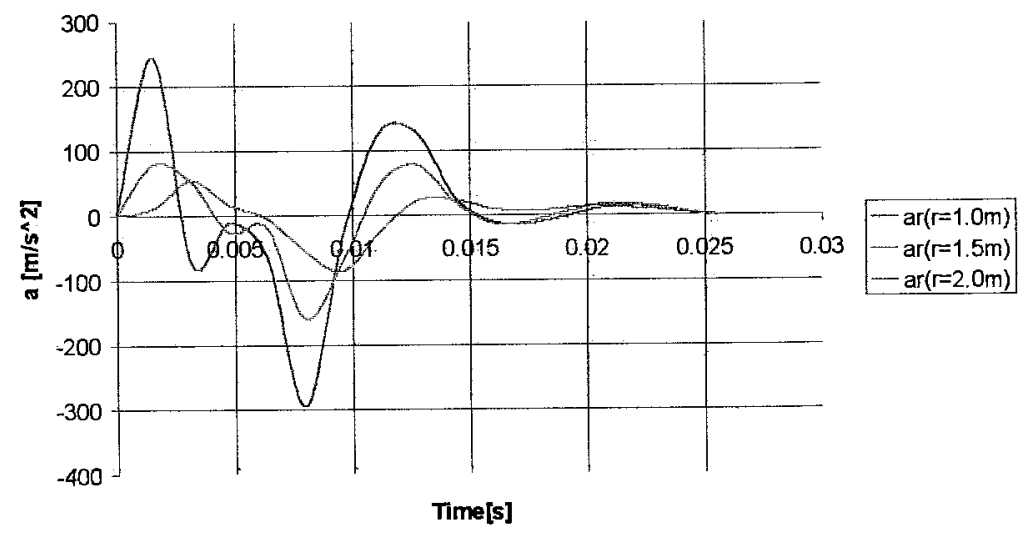

Figure 9 Vertical and Radial accelerations vs time, for the numerical simulation with $\mathrm{W}=1 \mathrm{~kg}$, explosive charge on the ground surface. 


\section{Surface profile}

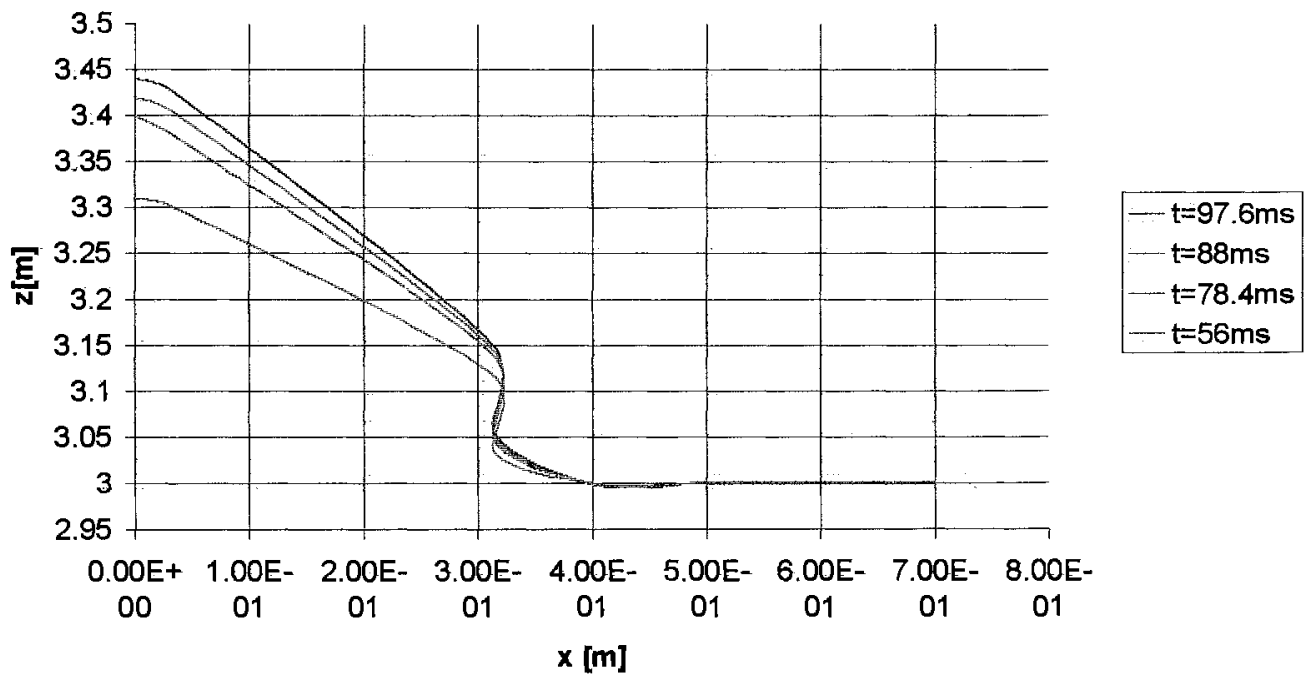

Figure 10 : Surface profile along radial plane for explosive charge on interface $(\mathrm{W}=1 \mathrm{~kg}$, ho:0.01 $\mathrm{m})$. Cleary showing that the crater radius is about $0.33 \mathrm{~m}$.

Crater diameter: Numerical vs. Experimental Results

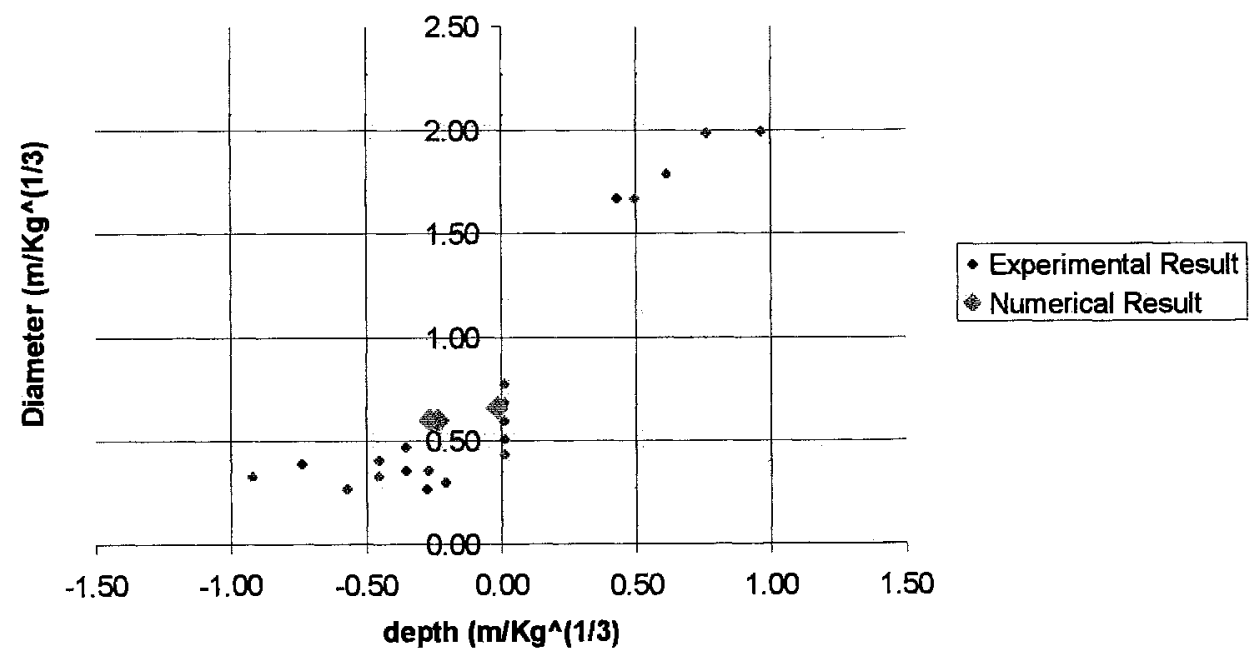

Figure 11: Comparison between numerical and experimental results 